\title{
On a spanning tree with specified leaves
}

\author{
Yoshimi Egawa
}

Department of Mathematical Information Science

Tokyo University of Science

1-3 Kagurazaka, Shinjuku-ku, Tokyo 162-8601 Japan

Haruhide Matsuda

The Research Institute of Educational Development, Tokai University

2-28-4 Tomigaya, Shibuya-ku, Tokyo, 151-8677 Japan

hmatsuda@ried.tokai.ac.jp

Tomoki Yamashita

Department of Mathematics, Asahi University

Hozumi, Gifu, 223-8522 Japan

tomoki@dent.asahi-u.ac.jp

Kiyoshi Yoshimoto

Department of Mathematics, College of Science and Technology

Nihon University

1-8 Kanda-Surugadai, Chiyoda-ku, Tokyo 101-8303 Japan

yosimoto@math.cst.nihon-u.ac.jp

August 7, 2006

\begin{abstract}
Let $k \geq 2$ be an integer. We show that if $G$ is a $(k+1)$-connected graph and each pair of nonadjacent vertices in $G$ has degree sum at least $|G|+1$, then for each subset $S$ of $V(G)$ with $|S|=k, G$ has a spanning tree such that $S$ is the set of endvertices. This result generalizes Ore's theorem which guarantees the existence of a Hamilton path connecting any two vertices.
\end{abstract}

Keywords: spanning tree; leaf connected; Hamilton path; Hamilton-connected 


\section{Introduction}

Many results concerning conditions for the existence of a Hamilton path are known. We can regard a Hamilton path as a spanning tree with precisely two endvertices. Thus it is natural to look for conditions which ensure the existence of a spanning tree with the bounded number of endvertices or with a specified set of endvertices. This paper is mainly concerned with sufficient conditions for a graph to have a spannning tree with a specified set of endvertices.

We consider finite undirected graphs without loops nor multiple edges. Let $G$ be a graph with vertex set $V(G)$ and edge set $E(G)$. The order of $G$ is denoted by $|G|$. For a vertex $x \in V(G)$, we denote the degree of $x$ in $G$ by $d_{G}(x)$ and the set of vertices adjacent to $x$ in $G$ by $N_{G}(x)$; thus $d_{G}(x)=\left|N_{G}(x)\right|$. For a subset $S \subset V(G)$, let $N_{G}(S)=\bigcup_{x \in S} N_{G}(x)$, and let $G-S$ denote the subgraph induced by $V(G) \backslash S$. A leaf (or an endvertex) of a tree is a vertex of degree one, and a branch vertex of a tree is a vertex of degree strictly greater than two. For a tree $T$, let

$$
\begin{aligned}
& L(T)=\{x \in V(T) \mid x \text { is a leaf of } T\} \text { and } \\
& B(T)=\{x \in V(T) \mid x \text { is a branch vertex of } T\} .
\end{aligned}
$$

A graph $G$ said to be $k$-leaf-connected if $|G|>k$ and for each subset $S$ of $V(G)$ with $|S|=k, G$ has a spanning tree $T$ with $L(T)=S$.

We prove the following theorem, which gives an Ore-type condition for a graph to be $k$-leaf-connected.

Theorem 1 Let $k \geq 2$ be an integer. Let $G$ be a $(k+1)$-connected graph and suppose that $d_{G}(x)+d_{G}(y) \geq|G|+1$ for any two nonajacent vertices $x, y \in V(G)$. Then $G$ is $k$-leaf-connected.

Theorem 1 is best possible in the following sense:

- We cannot replace the lower bound $|G|+1$ in the degree condition by $|G|$.

Consider the complete bipartite graph $G$ with partite sets $A$ and $B$ such that $|A|=|B|=t$, where $t$ is an integer with $t \geq k+1$. Then $G$ is $(k+1)$-connected, $|G|=2 t$, and $d_{G}(x)+d_{G}(y)=|G|$ for any two nonadjacent vertices $x$ and $y$ of $V(G)$. Suppose that $G$ is $k$-leaf-connected. Then $G$ has a spanning tree $T$ with $L(T) \subset B$. Consequently $d_{T}(x) \geq 2$ for all $x \in A$, and thus $|E(T)| \geq 2|A|=2 t$. However, this contradicts the fact $|E(T)|=|G|-1<2 t$. Hence $G$ is not $k$-leafconnected.

- For $k \geq 3$, the condition that $G$ is $(k+1)$-connected is necessary.

Assume that $k \geq 3$. Let $r \geq 1$ be an integer and consider the graph $G:=$ $K_{k}+\left(K_{1} \cup K_{r}\right)$. Then $G$ is $k$-connected but not $(k+1)$-connected, and for two vertices $x \in V\left(K_{r}\right)$ and $y \in V\left(K_{1}\right)$, we have $d_{G}(x)+d_{G}(y)=(|G|-2)+k \geq|G|+1$. 
However, $G$ has no spanning tree $T$ with $L(T)=V\left(K_{k}\right)$. (For the case where $k=2$, see Theorem 3 below and the first sentence in the paragraph following Theorem 3.)

As for the proof, we prove the following result, which is stronger than Theorem 1.

Theorem 2 Let $G$ be a graph, and let $S$ be a subset of $V(G)$ such that $|S| \geq 2, \mid N_{G}(S) \backslash$ $S \mid \geq 2, G-S$ is connected and $N_{G}(v) \backslash S \neq \emptyset$ for all $v \in S$. Suppose further that $d_{G}(x)+d_{G}(y) \geq|G|+1$ for any two nonajacent vertices $x, y \in V(G) \backslash S$. Then $G$ has a spanning tree $T$ with $L(T)=S$.

As in the case of Theorem 1, balanced complete bipartite graphs show that the lower bound in the degree condition in Theorem 2 is also sharp.

The following two results motivate our results. Since $G$ has a Hamilton path connecting any two vertices if and only if it is 2-leaf-connected, Theorem 1 is a natural extension of the following famous result.

Theorem 3 (Ore [2]) Let $G$ be a graph. If $d_{G}(x)+d_{G}(y) \geq|G|+1$ for every two nonajacent vertices $x, y \in V(G)$, then $G$ has a Hamilton path connecting any two vertices.

Note that if $d_{G}(x)+d_{G}(y) \geq|G|+k-1$ for every two nonajacent vertices $x, y \in V(G)$ then $G$ is $(k+1)$-connected. Thus the following result also follows from Theorem 1 (in [1], this result is derived from the assertion that the property of being $k$-leaf-connected is stable under a closure operation of Bondy-Chvátal type, i.e., if $x, y \in V(G)$ are nonadjacent vertices with $d_{G}(x)+d_{G}(y) \geq|G|+k-1$, then $G$ is $k$-leaf-connected if and only if $G+x y$ is $k$-leaf-connected; see [1; Theorem 4]).

Theorem 4 (Gurgel and Wakabayashi [1; Corollary 6.1]) Let $G$ be a graph, and suppose that $d_{G}(x)+d_{G}(y) \geq|G|+k-1$ for every two nonajacent vertices $x, y$ of $G$. Then $G$ is k-leaf-connected.

\section{Proof of Theorem 2}

Let $G$ and $S$ be as in Theorem 2. Since $N_{G}(v) \backslash S \neq \emptyset$ for each $v \in S$, and $G-S$ is connected, $G$ has a tree $T$ with $L(T)=S$ and $V(T) \backslash S \neq \emptyset$. Choose such a tree $T$ so that $|T|$ is as large as possible. If $V(G)=V(T)$, then we have nothing to prove. Thus we may assume that $G-V(T) \neq \emptyset$. Let $H$ be a component of $G-V(T)$ and set $X=N_{G}(H) \cap V(T)$. Note that $X \backslash S \neq \emptyset$ because $V(T) \backslash S \neq \emptyset$ and $G-S$ is connected.

We assume that we have chosen $H$ such that $|X|$ is as large as possible. We derive the proof into two cases according to the value of $|X|$.

Case 1. $|X|=1$.

Set $X=\left\{x_{0}\right\}$. Since $\left(N_{G}\left(H^{\prime}\right) \cap V(T)\right) \backslash S \neq \emptyset$ for every component $H^{\prime}$ of $G-V(T)$, it follows from our choice of $H$ that $N_{G}(G-V(T)) \cap S=\emptyset$, which implies $N_{G}(S) \subset V(T)$. 
Since $\left|N_{G}(S) \backslash S\right| \geq 2$ by the assumption of the theorem, we can take $v_{0} \in V(T) \backslash$ $\left(S \cup\left\{x_{0}\right\}\right)$. Now take $u_{0} \in V(H)$. By the assumption of Case $1, N_{G}\left(v_{0}\right) \cap N_{G}\left(u_{0}\right) \subset$ $\left\{x_{0}\right\}$. Since $v_{0} u_{0} \notin E(G)$, we also have $N_{G}\left(v_{0}\right) \cup N_{G}\left(u_{0}\right) \subset V(G) \backslash\left\{v_{0}, u_{0}\right\}$. Hence $d_{G}\left(v_{0}\right)+d_{G}\left(u_{0}\right) \leq|G|-2+1=|G|-1$, which contradicts the degree condition of the theorem. This completes the proof for Case 1 .

Case 2. $\quad|X| \geq 2$.

By the maximality of $T$, we obtain the following fact.

Fact $1 X$ is an indepedent set in $T$.

We denote by $P_{T}(a, b)$ the unique path in $T$ connecting two vertices $a$ and $b$ of $T$. We choose $x_{1} \in X \backslash S$ and $x_{2} \in X \backslash\left\{x_{1}\right\}$ so that $\left|P_{T}\left(x_{1}, x_{2}\right)\right|$ is as small as possible. By Fact $1, x_{1} x_{2} \notin E(T)$. We regard $T$ as an outdirected tree with root $x_{1}$. For $U \subset V(T)$, define $U^{+}=\bigcup_{u \in U}\left(N_{T}(u) \backslash V\left(P_{T}\left(x_{1}, u\right)\right)\right)$ and $U^{-}=\bigcup_{u \in U}\left(N_{T}(u) \cap V\left(P_{T}\left(x_{1}, u\right)\right)\right)$. For a vertex $u \in V(T) \backslash\left\{x_{1}\right\}$, having in mind the fact that $\left|\{u\}^{-}\right|=1$, we let $u^{-}$denote the unique vertex in $\{u\}^{-}$. Recall that $B(T)$ denotes the set of branch vertices of $T$.

Claim $2 B(T)^{+} \cap X=\emptyset$.

Proof. Suppose that $x \in B(T)^{+} \cap X$. Let $x^{\prime} \in N_{G}(x) \cap V(H)$ and $x_{1}^{\prime} \in N_{G}\left(x_{1}\right) \cap V(H)$, and let $Q$ be a path in $H$ connecting $x^{\prime}$ and $x_{1}^{\prime}$. Then $T^{\prime}:=\left(T-x x^{-}+x x^{\prime}+x_{1} x_{1}^{\prime}\right) \cup Q$ is a tree with $L\left(T^{\prime}\right)=S$ and $\left|T^{\prime}\right|>|T|$. This contradicts the maximality of $T$. Hence $B(T)^{+} \cap X=\emptyset$.

Set $W=B(T) \cup\left\{x_{1}\right\}$. Choose $y_{1} \in\left(V\left(P_{T}\left(x_{1}, x_{2}\right)\right) \cap W\right) \backslash\left\{x_{2}\right\}$ so that $\left|P_{T}\left(y_{1}, x_{2}\right)\right|$ is as small as possible (possibly $y_{1}=x_{1}$ ). By Claim 2, $y_{1} x_{2} \notin E(T)$. Write $N_{T}\left(y_{1}\right) \cap$ $V\left(P_{T}\left(y_{1}, x_{2}\right)\right)=\left\{v_{1}\right\}$ and $N_{T}\left(x_{2}\right) \cap V\left(P_{T}\left(y_{1}, x_{2}\right)\right)=\left\{v_{2}\right\}$ (possibly $\left.v_{1}=v_{2}\right)$. Write $N_{T}\left(x_{1}\right) \cap V\left(P_{T}\left(x_{1}, x_{2}\right)\right)=\left\{w_{1}\right\}$ and define $T^{*}=T-V\left(P_{T}\left(w_{1}, v_{2}\right)\right)$. We denote by $P_{1}, P_{2}, \ldots, P_{m}$ the components of $T^{*}-\left\{u v \in E(T) \mid u \in W, v \in\{u\}^{+}\right\}$. We may assume that $V\left(P_{1}\right)=\left\{x_{1}\right\}$ and $x_{2} \in V\left(P_{2}\right)$. Note that $P_{i}$ is a path for every $i=1, \ldots, m$ and $\left|V\left(P_{i}\right) \cap W^{+}\right|=1$ for each $i=3, \ldots, m$. Write $V\left(P_{i}\right) \cap W^{+}=\left\{a_{i}\right\}$ for each $i=3, \ldots, m$. Then for each $i, a_{i}$ is an endvertex of $P_{i}$.

For $j=1,2$, let $u_{j} \in N_{G}\left(x_{j}\right) \cap V(H)$ (possibly $u_{1}=u_{2}$ ).

Claim $3\left|N_{G}\left(u_{1}\right) \cap V\left(T^{*}\right)\right|+\left|N_{G}\left(u_{2}\right) \cap V\left(T^{*}\right)\right| \leq\left|T^{*}\right|+2$.

Proof. Since $\left|P_{1}\right|=\left|\left\{x_{1}\right\}\right|=1,\left|N_{G}\left(u_{1}\right) \cap V\left(P_{1}\right)\right|+\left|N_{G}\left(u_{2}\right) \cap V\left(P_{1}\right)\right| \leq 2=\left|P_{1}\right|+1$.

By Fact $1,\left(N_{G}\left(u_{1}\right) \cap V\left(P_{i}\right)\right)^{-} \cap\left(N_{G}\left(u_{2}\right) \cap V\left(P_{i}\right)\right)=\emptyset$ for every $2 \leq i \leq m$. For the path $P_{2}$, we have $\left|N_{G}\left(u_{1}\right) \cap V\left(P_{2}\right)\right|=\left|\left(N_{G}\left(u_{1}\right) \cap V\left(P_{2}\right)\right)^{-}\right|$and $\left(N_{G}\left(u_{1}\right) \cap V\left(P_{2}\right)\right)^{-} \cup$ $\left(N_{G}\left(u_{2}\right) \cap V\left(P_{2}\right)\right) \subset V\left(P_{2}\right) \cup\left\{v_{2}\right\}$. Hence $\left|N_{G}\left(u_{1}\right) \cap V\left(P_{2}\right)\right|+\left|N_{G}\left(u_{2}\right) \cap V\left(P_{2}\right)\right|=$ $\left|\left(N_{G}\left(u_{1}\right) \cap V\left(P_{2}\right)\right)^{-}\right|+\left|N_{G}\left(u_{2}\right) \cap V\left(P_{2}\right)\right| \leq\left|P_{2}\right|+1$. Let now $3 \leq i \leq m$. Then $a_{i} \notin N_{G}\left(u_{1}\right)$ by Fact 1 or Claim 2 according as $a_{i} \in\left\{x_{1}\right\}^{+}$or $a_{i} \in B(T)^{+}$. Since $u^{-} \in V\left(P_{i}\right)$ for all $u \in V\left(P_{i}\right) \backslash\left\{a_{i}\right\}$, this implies $\left(N_{G}\left(u_{1}\right) \cap V\left(P_{i}\right)\right)^{-} \cup\left(N_{G}\left(u_{2}\right) \cap V\left(P_{i}\right)\right) \subset V\left(P_{i}\right)$. Since $\left|\left(N_{G}\left(u_{1}\right) \cap V\left(P_{i}\right)\right)^{-}\right|=\left|N_{G}\left(u_{1}\right) \cap V\left(P_{i}\right)\right|$, we obtain $\left|N_{G}\left(u_{1}\right) \cap V\left(P_{i}\right)\right|+\left|N_{G}\left(u_{2}\right) \cap V\left(P_{i}\right)\right| \leq$ 
$\left|\left(N_{G}\left(u_{1}\right) \cap V\left(P_{i}\right)\right)^{-}\right|+\left|N_{G}\left(u_{2}\right) \cap V\left(P_{i}\right)\right| \leq\left|P_{i}\right|$. Thus $\left|\left(N_{G}\left(u_{1}\right) \cap V\left(P_{i}\right)\right)\right|+\mid N_{G}\left(u_{2}\right) \cap$ $V\left(P_{i}\right)|\leq| P_{i} \mid$ for every $3 \leq i \leq m$. Consequently

$$
\begin{aligned}
\left|N_{G}\left(u_{1}\right) \cap V\left(T^{*}\right)\right|+\left|N_{G}\left(u_{2}\right) \cap V\left(T^{*}\right)\right| & =\sum_{i=1}^{m}\left(\left|N_{G}\left(u_{1}\right) \cap V\left(P_{i}\right)\right|+\left|N_{G}\left(u_{2}\right) \cap V\left(P_{i}\right)\right|\right) \\
& \leq\left|P_{1}\right|+1+\left|P_{2}\right|+1+\sum_{i=3}^{m}\left|P_{i}\right| \\
& =\left|T^{*}\right|+2 .
\end{aligned}
$$

Hence the claim holds.

Let $R$ be a path in $H$ connecting $u_{1}$ and $u_{2}$.

Claim $4\left|N_{G}\left(v_{1}\right) \cap V\left(T^{*}\right)\right|+\left|N_{G}\left(v_{2}\right) \cap V\left(T^{*}\right)\right| \leq\left|T^{*}\right|+2$.

Proof. Note that $\left|N_{G}\left(v_{1}\right) \cap V\left(P_{1}\right)\right|+\left|N_{G}\left(v_{2}\right) \cap V\left(P_{1}\right)\right| \leq 2=\left|P_{1}\right|+1$. Note also that $\left(N_{G}\left(v_{1}\right) \cap V\left(P_{2}\right)\right) \cup\left(N_{G}\left(v_{2}\right) \cap V\left(P_{2}\right)\right)^{-} \subset V\left(P_{2}\right) \cup\left\{v_{2}\right\}$. We now show $a_{i} \notin N_{G}\left(v_{2}\right)$ for every $i=3, \ldots, m$. Suppose that $a_{j} \in N_{G}\left(v_{2}\right)$ for some $j$ with $3 \leq j \leq m$. Then $T^{\prime}:=\left(T-a_{j} a_{j}^{-}-v_{2} x_{2}+a_{j} v_{2}+x_{1} u_{1}+x_{2} u_{2}\right) \cup R$ is a tree with $L\left(T^{\prime}\right)=S$ and $\left|T^{\prime}\right|>|T|$. But this contradicts the maximality of $T$. Hence $a_{i} \notin N_{G}\left(v_{2}\right)$ for every $i=3, \ldots, m$. Consequently, $\left(N_{G}\left(v_{1}\right) \cap V\left(P_{i}\right)\right) \cup\left(N_{G}\left(v_{2}\right) \cap V\left(P_{i}\right)\right)^{-} \subset V\left(P_{i}\right)$ for each $i=3, \ldots, m$.

Next, suppose that $\left(N_{G}\left(v_{1}\right) \cap V\left(P_{j}\right)\right) \cap\left(N_{G}\left(v_{2}\right) \cap V\left(P_{j}\right)\right)^{-} \neq \emptyset$ for some $j$ with $2 \leq j \leq$ $m$. Then there exists $v \in V\left(P_{j}\right)$ such that $v \in N_{G}\left(v_{2}\right) \cap V\left(P_{j}\right)$ and $v^{-} \in N_{G}\left(v_{1}\right) \cap V\left(P_{j}\right)$. But then $T^{\prime}:=\left(T-v v^{-}-v_{1} y_{1}-v_{2} x_{2}+v_{2} v+v_{1} v^{-}+x_{1} u_{1}+x_{2} u_{2}\right) \cup R$ is a tree with $L\left(T^{\prime}\right)=S$ and $\left|T^{\prime}\right|>|T|$, which is a contradiction. Hence $\left(N_{G}\left(v_{1}\right) \cap V\left(P_{i}\right)\right) \cap\left(N_{G}\left(v_{2}\right) \cap V\left(P_{i}\right)\right)^{-}=\emptyset$ for each $i=2, \ldots, m$.

Since $\left|\left(N_{G}\left(v_{2}\right) \cap V\left(P_{i}\right)\right)^{-}\right|=\left|N_{G}\left(v_{2}\right) \cap V\left(P_{i}\right)\right|$ for every $2 \leq i \leq m$, we obtain $\left|N_{G}\left(v_{1}\right) \cap V\left(P_{2}\right)\right|+\left|N_{G}\left(v_{2}\right) \cap V\left(P_{2}\right)\right| \leq\left|P_{2}\right|+1$ and $\left|N_{G}\left(v_{1}\right) \cap V\left(P_{i}\right)\right|+\left|N_{G}\left(v_{2}\right) \cap V\left(P_{i}\right)\right| \leq$ $\left|P_{i}\right|$ for every $3 \leq i \leq m$. Therefore $\left|N_{G}\left(v_{1}\right) \cap V\left(T^{*}\right)\right|+\left|N_{G}\left(v_{2}\right) \cap V\left(T^{*}\right)\right| \leq\left|T^{*}\right|+2$.

Now let $j \in\{1,2\}$. By the minimality of $\left|P_{T}\left(x_{1}, x_{2}\right)\right|$, we have $u_{j} v_{j} \notin E(G)$. Note that $u_{j}, v_{j} \notin S$ because $u_{j} \notin V(T)$ and $v_{j} \in V\left(P_{T}\left(x_{1}, x_{2}\right)\right) \backslash\left\{x_{1}, x_{2}\right\}$. Thus by the degree condition, $d_{G}\left(u_{j}\right)+d_{G}\left(v_{j}\right) \geq|G|+1$. Furthermore, by the choice of $x_{1}$ and $x_{2}, N_{G}\left(v_{j}\right) \cap$ $V(H)=\emptyset$ and $N_{G}\left(u_{j}\right) \cap V\left(P_{T}\left(w_{1}, v_{2}\right)\right)=\emptyset$. Since we clearly have $N_{G}\left(u_{j}\right) \cap(V(G) \backslash$ $(V(T) \cup V(H)))=\emptyset, N_{G}\left(u_{j}\right) \cap V(H) \subset V(H) \backslash\left\{u_{j}\right\}$ and $N_{G}\left(v_{j}\right) \cap V\left(P_{T}\left(w_{1}, w_{2}\right)\right) \subset$ $V\left(P_{T}\left(w_{1}, v_{2}\right)\right) \backslash\left\{v_{j}\right\}$, this implies

$$
\begin{aligned}
& \left|N_{G}\left(u_{j}\right) \cap\left(V(G) \backslash V\left(T^{*}\right)\right)\right|+\left|N_{G}\left(v_{j}\right) \cap\left(V(G) \backslash V\left(T^{*}\right)\right)\right| \\
& \leq|G-(V(T) \cup V(H))|+(|H|-1)+\left(\left|P_{T}\left(w_{1}, v_{2}\right)\right|-1\right)=|G|-\left|T^{*}\right|-2 .
\end{aligned}
$$

Consequently

$$
\left|N_{G}\left(u_{j}\right) \cap V\left(T^{*}\right)\right|+\left|N_{G}\left(v_{j}\right) \cap V\left(T^{*}\right)\right| \geq|G|+1-\left(|G|-\left|T^{*}\right|-2\right)=\left|T^{*}\right|+3 .
$$


Thus $\left|N_{G}\left(u_{j}\right) \cap V\left(T^{*}\right)\right|+\left|N_{G}\left(v_{j}\right) \cap V\left(T^{*}\right)\right| \geq\left|T^{*}\right|+3$ for each $j=1,2$. This implies that we have $\left|N_{G}\left(u_{1}\right) \cap V\left(T^{*}\right)\right|+\left|N_{G}\left(u_{2}\right) \cap V\left(T^{*}\right)\right| \geq\left|T^{*}\right|+3$ or $\left|N_{G}\left(v_{1}\right) \cap V\left(T^{*}\right)\right|+$ $\left|N_{G}\left(v_{2}\right) \cap V\left(T^{*}\right)\right| \geq\left|T^{*}\right|+3$, which contradicts Claim 3 or 4 .

This completes the proof of Theorem 2 .

\section{Application}

As a consequence of Theorem 1, we prove the following result, which gurantees the existence of a spanning tree having the bounded number of leaves and containing specified vertices as leaves.

Corollary 5 Let $k$ and $s$ be integers with $k \geq 2$ and $0 \leq s \leq k$. Suppose that $G$ is an $(s+1)$-connected graph, and for any two nonadjacent vertices $x, y \in V(G)$,

$$
d_{G}(x)+d_{G}(y) \geq|G|-k+1+s .
$$

Then for any subset $S \subset V(G)$ with $|S|=s, G$ has a spanning tree $T$ such that $S \subset L(T)$ and $|L(T)| \leq k$.

Proof. Construct a new graph $H$ by joining two graphs $G$ and $K_{k-s}$. Then $H$ satisfies the conditions of Theorem 1, and hence $H$ has a spanning tree $T$ such that $L(T)=$ $S \cup V\left(K_{k-s}\right)$. Thus $T-V\left(K_{k-s}\right)$ is a spanning tree of $G$ with the desired properties.

In Corollary 5 , the lower bound in the degree condition is sharp. For example, let $G$ be a complete bipartite graph with partite sets $A$ and $B$ such that $|A|=t+k$ and $|B|=t+s$, where $t \geq 1$. Then $G$ is $(s+1)$-connected, $|G|=2 t+k+s$, and $d_{G}(x)+d_{G}(y) \geq 2|B|=2 t+2 s=|G|-k+s$ for any two nonadjacent vertices $x$ and $y$ of $G$. Suppose that $G$ has a spanning tree $T$ such that $|L(T)| \leq k$ and $s$ specified vertices in $B$ are contained in $L(T)$. Then the number of edges in $T$ is at least $2|A|-(k-s)=2 t+k+s$. However, this is a contradiction because $2 t+k+s>|G|-1=|E(T)|$. Thus $G$ has no desired spanning tree.

Moreover, for $k \geq 3$ and $s \geq 1$, the condition that $G$ is $(s+1)$-connected is necessary. Assume that $k \geq 3$. Let $r \geq 1$, and consider the graph $G:=K_{s}+\left(K_{1} \cup K_{r}\right)$. Then $G$ is $s$-connected but not $(s+1)$-connected. For $x \in V\left(K_{1}\right)$ and any $y \in V\left(K_{r}\right)$, we have $d_{G}(x)+d_{G}(y)=(|G|-2)+s \geq|G|-k+1+s$. However, $G$ has no spanning tree $T$ with $V\left(K_{s}\right) \subseteq L(T)$.

\section{References}

[1] M.A. Gurgel and Y. Wakabayashi, On k-leaf-connected graphs, J. Combin. Theory Ser. B 41 (1986) 1-16.

[2] O. Ore, Hamilton connected graphs, J. Math. Pures Appl. 42 (1963) 21-27. 